Veritas E Scientia

Vol. 8. $\mathrm{N}^{\circ} 1$.

Enero - Junio del 2019.

ISSN Edición Online: 2617-0639

https://doi.org/10.47796/ves.v8i1.114

\title{
DESARROLLAR UNA METODOLOGÍA DE IMPLEMENTACIÓN DE LA NORMA NTP-ISO/IEC 17025:2017 PARA LA ACREDITACIÓN DE LABORATORIOS DE ENSAYO DE SUELOS, CONCRETOS Y PAVIMENTOS EN UNIVERSIDADES PRIVADAS DEL PERÚ - 2018
}

\author{
DEVELOP AN IMPLEMENTATION METHODOLOGY OF THE NTP-ISO / IEC 17025: 2017 STANDARD \\ FOR THE ACCREDITATION OF SOIL, CONCRETE AND PAVEMENT TEST LABORATORIES IN PRIVATE \\ UNIVERSITIES OF PERU - 2018
}

\author{
María Etelvina Duarte Lizarzaburu ${ }^{1}$ \\ José Antonio Salgado Canal ${ }^{2}$ \\ Presentado: $27 / 10 / 2018$ \\ Aceptado: $17 / 06 / 2019$ \\ Publicado online:17/07/2019
}

\section{RESUMEN}

El propósito fue desarrollar una metodología de implementación de la Norma NTP ISO/IEC 17025:2017 para la acreditación de laboratorios de ensayo de suelos, concretos y pavimentos en universidades privadas del Perú, para lo cual se utilizó como marco de referencia el PMBOK y los requisitos de la Norma NTP ISO/IEC 17025:2017.Se estructuró la metodología en tres componentes principales: diagnóstico, gestión, operación y mantenimiento, cada uno de los cuales contiene un grupo de formatos estructurados en función a los requisitos normativos a implementarse. La metodología propuesta es una herramienta de ayuda a laboratorios universitarios que aspiran acreditarse, que garanticen la calidad y confiabilidad de los ensayos y coadyuven a su gestión como centro de formación, investigación y de servicio. En el Perú son escasos los laboratorios acreditados de universidades, problema que se refleja en el posicionamiento de nuestro país en cuanto a calidad en infraestructura, en tal sentido, la metodología propuesta sería de mucha ayuda en el proceso de acreditación de laboratorios y por ende mejorar la calidad de la infraestructura.

Palabras Clave: Acreditación, calidad, Norma NTP ISO IEC/17025:2017, PMBOK, INACAL.

\section{ABSTRACT}

The purpose was to develop a methodology for the implementation of Standard NTP ISO / IEC 17025: 2017 for the accreditation of soil, concrete

\footnotetext{
${ }^{1}$ Magíster en Ingeniería Civil con mención en Gerencia de la Construcción

2 Magíster, Coordinador de Investigación de pregrado de la Facultad de Ingeniería Civil de Universidad Nacional de Ingeniería. Perú.
} 
and pavement testing laboratories in private universities in Peru, for which the PMBOK and the requirements of Standard NTP ISO / IEC 17025: 2017. The methodology was structured into three main components: diagnosis, management, operation and maintenance, each of which contains a group of formats structured according to the regulatory requirements to be implemented. The proposed methodology is a tool to help university laboratories that aspire to be accredited, that guarantee the quality and reliability of the tests and contribute to their management as a training, research and service center. In Peru there are few accredited laboratories of universities, a problem that is reflected in the positioning of our country in terms of quality infrastructure, in this sense, the proposed methodology would be very helpful in the process of accreditation of laboratories and therefore improve the quality of the infrastructure.

Keywords: Accreditation, Quality, Norma NTP ISO IEC / 17025: 2017, PMBOK, INACAL.

\section{INTRODUCCIÓN}

Los laboratorios universitarios tienen por principio orientarse al cumplimiento de su responsabilidad como espacios de formación e investigación. Por ello la importancia de desarrollar una metodología de implementación de la Norma NTP ISO/IEC 17025:2017, cuyo cumplimiento posibilita que cualquier laboratorio demuestre que opera de forma competente, imparcial y que tiene la capacidad de generar resultados válidos. Para los laboratorios de ensayo e investigación universitarios puede utilizarse como una herramienta para satisfacer las necesidades de las partes interesadas (investigadores, estudiantes, patrocinadores y clientes), el cual sea de fácil comprensión e implementación y que permita garantizar la confiabilidad de los resultados obtenidos. El propósito del presente trabajo es plasmar el diseño de una metodología integrando los aportes del PMI (Project Management Institute) a través del PM BOK y los requisitos de la Norma NTP ISO/IEC 17025:2017 para finalmente, proceder a validar la propuesta con expertos, evidenciando de esta manera que la metodología puede coadyuvar a los laboratorios universitarios a alcanzar la acreditación y otorgarles la seguridad y la confianza en los resultados generados.

El Foro Económico Mundial WEF (World Economic Fórum) publicó el Informe Global de Competitividad (ICG) 2017-2018 que evalúa los factores que impulsan la productividad y crecimiento en 137 países. El Perú se ubica en el puesto 72 del ICG (Índice de competitividad global), lo cual representa un retroceso de 5 posiciones respecto al Informe del año anterior y 11 posiciones respecto al puesto 61 que ocupó el año 2013. El país aún no ha avanzado lo esperado en relación a la calidad de infraestructura, que es uno de los pilares para analizar la competitividad y el crecimiento de las economías mundiales. Para el periodo 20172018, el Perú se ubica en el puesto 86 a nivel mundial con respecto a la calidad de la infraestructura (Informe Global de Competitividad - ICG del World Economic Forum 2017-2018), se encuentra por detrás de varios países de la región y muy por debajo de los países mejor ubicados en este ranking. El modelo económico de la competitividad del WEF considera que los países pasan por tres fases de desarrollo con 2 fases de transición. Primera Fase: Economía basada en factores básicos de producción. Segunda fase: Economía basada en la eficiencia (nuestro país se encuentra ubicado en esta fase). Tercera Fase: Economía basada en la Innovación. Uno de los requisitos más importantes para asegurar la calidad de la infraestructura y formalizar los procesos de control en la ejecución de obras es contratar un laboratorio que preste un trabajo especializado, el cliente busca calidad, confiabilidad, disponibilidad y un servicio sin 
errores. Tomando como referencia información obtenida de SUNAT y del OSCE (Organismo Supervisor de las Contrataciones del Estado) para identificar las empresas que cuentan con Registro Nacional de Proveedores - RNP que ofrecen servicios de ensayos de laboratorio vinculados al control de calidad en el rubro de la construcción y la ingeniería civil, se determinó que actualmente en el Perú existen poco más de cien proveedores que realizan este tipo de servicios. De otro lado, según el Directorio de Organismos Acreditados del INACAL y reportes vigentes a julio del 2018, en el Perú existen sólo 90 laboratorios acreditados en diversos rubros (microbiológicos, hidrobiológicos, químicos, físicos, mecánicos, textiles, entre otros). De estos 90 laboratorios, sólo seis corresponden a laboratorios de Universidades: Univ. Cayetano Heredia (Lima), Pontificia Univ. Católica del Perú (Lima), Univ. Nacional La Molina (en Lima), Univ. De Piura, Univ. Santiago Antúnez de Mayolo (Ancash), Univ. Católica Santa María (Arequipa). Sólo el laboratorio de la Pontificia Univ. Católica realiza ensayos en el rubro de la construcción. Así mismo, de los 90 laboratorios acreditados, se identificó que sólo seis laboratorios realizan ensayos vinculados a la ingeniería civil y la construcción.

En la actualidad son muy pocos los laboratorios de ensayo de universidades privadas del Perú que se encuentran acreditados bajo la Norma NTP ISO/IEC 17025. Ejecutar un proyecto bajo parámetros de calidad, redunda en una buena inversión, mejora la productividad, impulsa el crecimiento y posicionamiento económico del país y por ende se orienta a optimizar la calidad de vida de la población.

La rigurosa competencia entre las organizaciones que prestan servicios de educación superior y el rápido crecimiento y proliferación de estas entidades, ha conllevado a exigir altos niveles de calidad en el campo en el que estas se especializan, para ser altamente competentes en un medio de gran exigencia y demanda (Contreras y Galleguillos,2009). Existen experiencias al respecto, donde Córdova, M.(2012), ya define el grado de implementación en base a un diagnóstico situacional del LEM y propone los procesos que se deben realizarse para adecuarse a los requisitos normativos. Tejerina, M. (2010), brinda una visión general de la situación actual del laboratorio con la perspectiva de mejorar su sistema de gestión en base a la Norma NTP-ISO/IEC 17025. Díaz, M., \& Araya, M. (2007), adapta y genera la documentación necesaria para que un área determinada cumpla los estándares de la ISO 17025.

Hablar de calidad es de suma importancia, pues no sólo se trata de la calidad de un servicio que ofrece un laboratorio, éste se proyecta a una construcción de calidad y al uso de materiales de buena calidad. Para entender el origen de la normalización es importante familiarizarse con las organizaciones internacionales que las reglamentan y que está conformada por diversos estamentos representativos de cada país miembro como son ISO (International Organization for Standardization), IEC (International Electrotechnical Commission), IAAC (Cooperación Inter-americana de Acreditación), IAF (Foro Internacional de Acreditación), ILAC (Cooperación Internacional de Acreditación de Laboratorios), CEN (Comité Europeo de Normalización). En el Perú, el INACAL (Instituto Nacional de Calidad) sujeta al marco normativo y actuará en concordancia con la política, los planes y los objetivos que apruebe el Consejo Nacional para la Calidad.

El sistema de acreditación del Organismo de Acreditación, OAE, ofrece a los interesados en la acreditación, un procedimiento donde están definidos los requisitos de que deben cumplir para su acreditación. Llanos, P.(2013). Es importante para el comprador, el ente regulador y el público saber que los Organismos de Evaluación de la Conformidad-OEC son competentes para desempeñar sus tareas. López, P. (2015), Cómo documentar un sistema de gestión de calidad según ISO 9001:2015. La ISO 9001:2015 es la versión vigente de esta Norma, éste texto es una guía práctica y clara que puede ser utilizada por personas que están involucradas en gestión de la calidad pero que no son necesariamente expertos en la norma. Los laboratorios que implementen la Norma NTP-ISO/IEC 17025, también están obligados a usar la Norma ISO 9001. 


\section{OBJETIVOS}

a) Desarrollar un diagnóstico situacional que nos permita analizar el grado de implementación de la Norma NTP-ISO/IEC 17025:2017 en laboratorios de ensayo de suelos, concretos y pavimentos de Universidades Privadas del Perú.

b) Elaborar los procesos y procedimientos de las componentes de la metodología de implementación para la acreditación de laboratorios de ensayo conforme a los requisitos que exige la Norma NTPISO/IEC 17025:2017.

c) Validar la metodología de implementación de la Norma NTP ISO/IEC 17025:2017 para la acreditación de laboratorios propuesta.

\section{METODOLOGÍA}

Investigación aplicada basada en la modalidad de innovación para la aplicación de un Plan de implementación de la Norma NTP ISO/IEC 17025:2017. La población de estudio para el presente trabajo está representada por los encargados de los laboratorios de las Universidades Privadas existentes en el sur del Perú en las cuales se dicta la carrera de Ing. Civil y por consultores especialistas en la Norma NTP ISO/IEC 17025:2017. La muestra se orienta a los laboratorios de suelos, concretos y pavimentos de universidades privadas ubicados en la zona sur del Perú. Se aplicó la técnica de la encuesta cuyas unidades de observación fueron los ingenieros que laboran como encargados de laboratorios de suelos, concretos y pavimentos de universidades privadas.

\section{RESULTADOS}

Fue importante la aplicación de la encuesta y las conclusiones derivadas de esta, ya que se ratificó la ausencia de laboratorios universitarios acreditados y la necesidad de implementar una metodología que ayude a mejorar muchos aspectos relativos a la calidad del servicio que ofrecen los laboratorios. Se pudo observar que, de la muestra, el $90 \%$ son ingenieros civiles y un $10 \%$ ejercen otras profesiones afines y que pueden desenvolverse en un laboratorio. El 50\% tienen una edad superior a los 40 años. El 60\% tienen más de 3 años de experiencia como jefes de laboratorio, el 40\% restante cuenta con 1 a 3 años. El 80\% conocen el problema que supone el no contar con un laboratorio acreditado. El $70 \%$ no han sido capacitados en la Norma ISO 17025. El 60\% de los laboratorios no se encuentran debidamente implementados (ambientes y equipamiento adecuado). El 70\% no cuenta con normas originales para desarrollar sus ensayos. El $80 \%$ no cuenta con manuales ni políticas de calidad. El 60\% de universidades no capacita a su personal. El $70 \%$ de los laboratorios cuenta con menos de 5 trabajadores involucrados al trabajo de laboratorio.

Definición de la propuesta.- El modelo de propuesta está aplicado a una metodología que tiene por finalidad promover el desarrollo de los procesos para la implementación de la Norma NTP ISO/IEC 17025:2017, para la acreditación de laboratorios de ensayo, tomando como referencia la guía de los Fundamentos para la dirección de Proyectos, el PMBOK. 

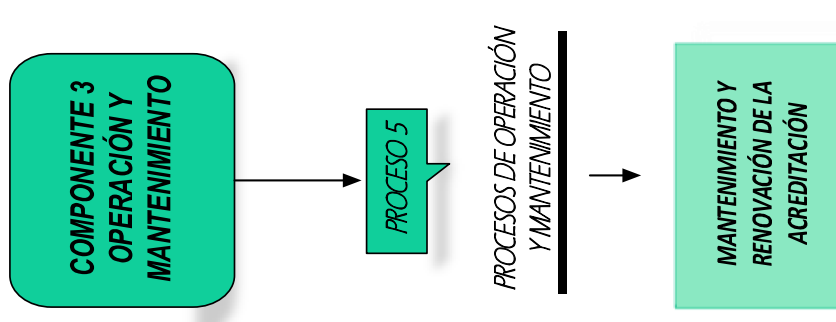

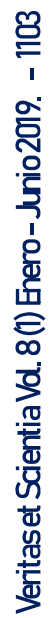

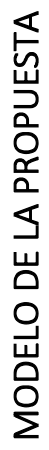

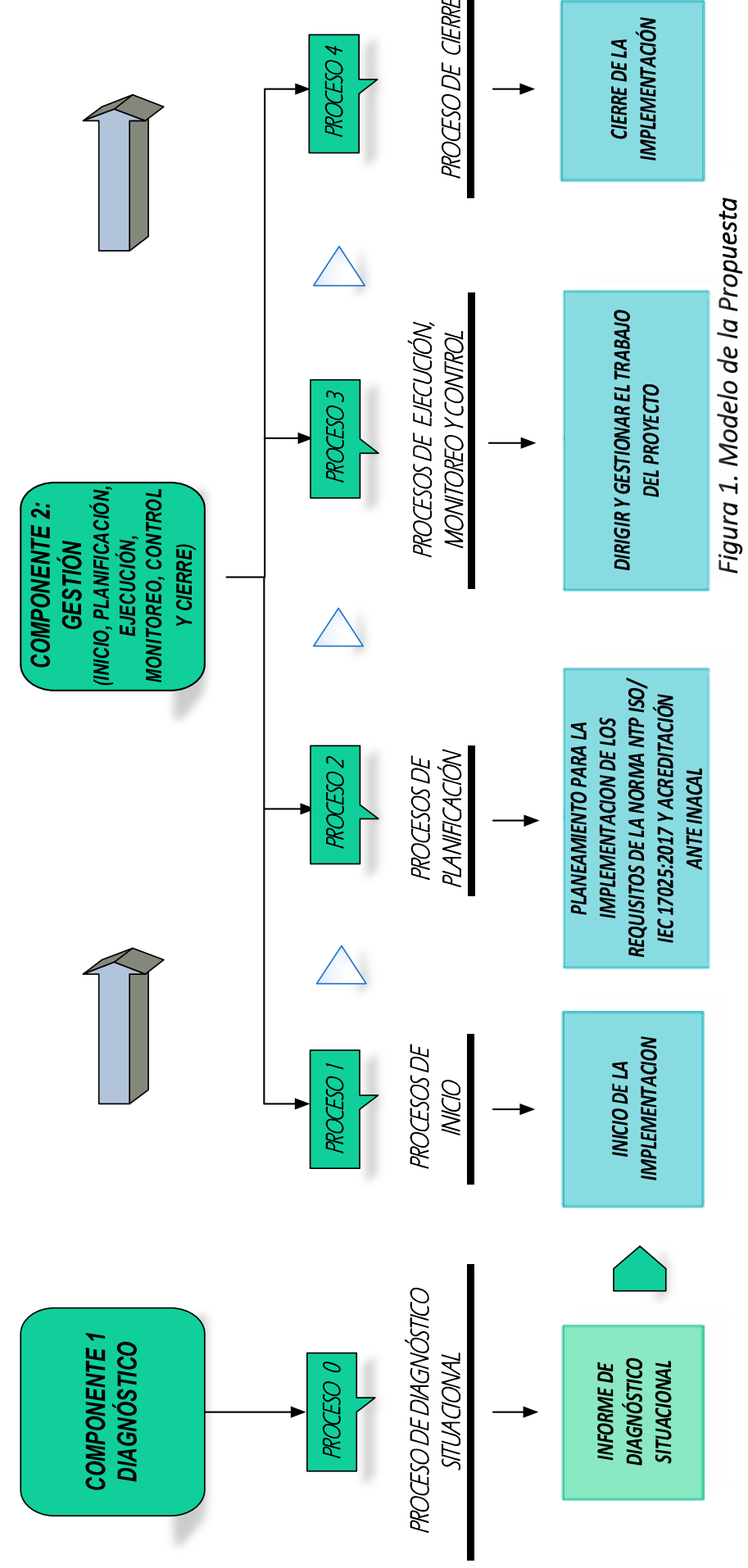




\section{COMPONENTE 1: DIAGNÓSTICO}

El diagnóstico tiene por finalidad identificar un conjunto de factores internos y externos relacionados con el laboratorio y que inciden en el desarrollo del mismo; de esta forma se podrá determinar si es viable alcanzar la acreditación conforme a los requisitos de la Norma NTP ISO/IEC 17025:2017. (Fig. 2)

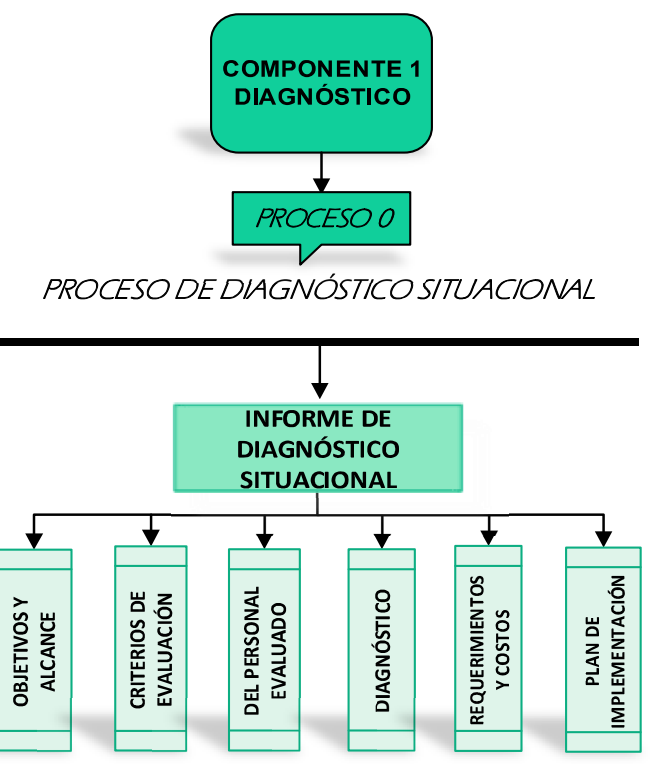

Figura 2. Componente 1: Diagnóstico

\section{COMPONENTE 2: GESTIÓN}

El Componente 2 del modelo propuesto está estructurado en función de los principales grupos de procesos, del PMBOK como: inicio, planificación, ejecución, monitoreo, control y cierre, así como también en base a los requisitos de la Norma NTP ISO/IEC 17025:2017. (Fig. 3)

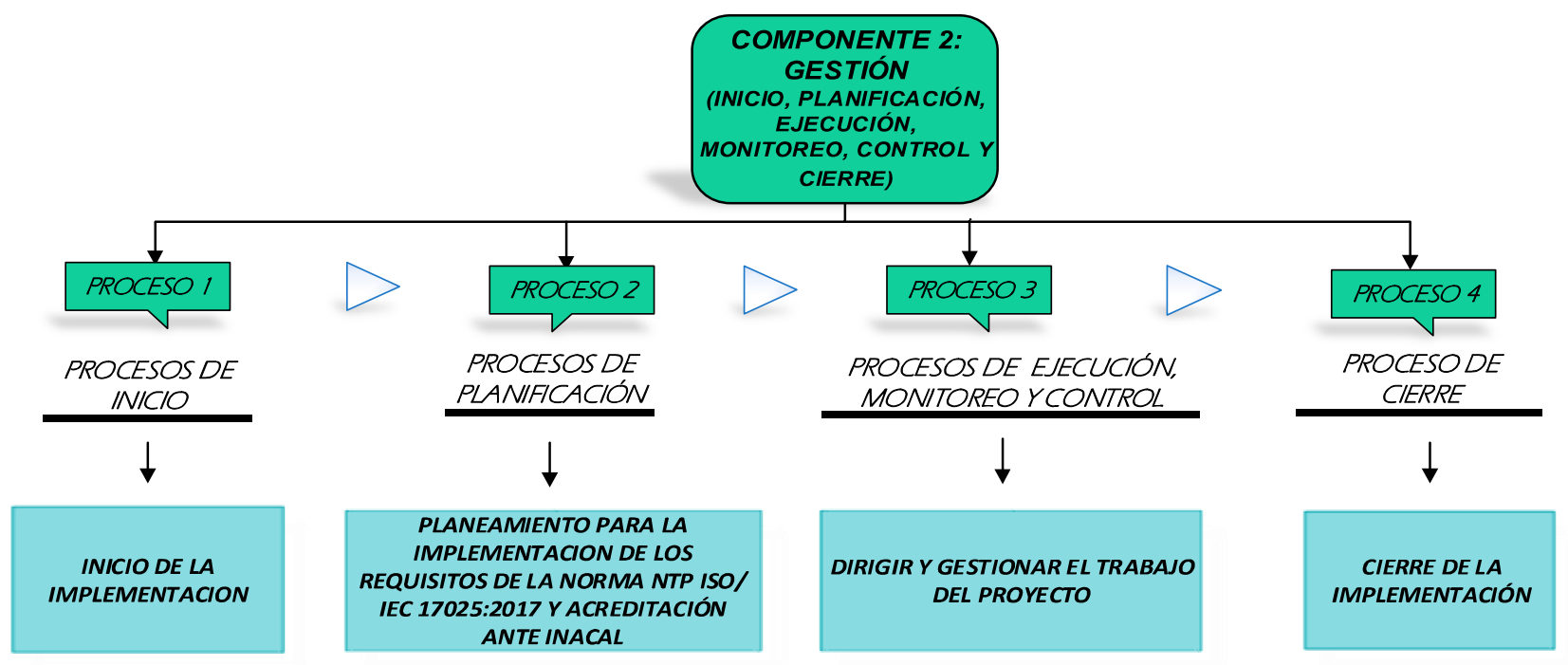

Figura 2. Componente 3: Gestión 


\section{COMPONENTE 3: OPERACIÓN Y MANTENIMIENTO}

Esta etapa se desarrolla a partir de la obtención del Certificado de Acreditación y se mantiene durante el periodo de vigencia de la acreditación. Se garantizará que la implementación de los requisitos de la Norma NTP ISO/IEC 17025:2017 haya producido una mejora en la calidad de los servicios que ofrece el laboratorio.

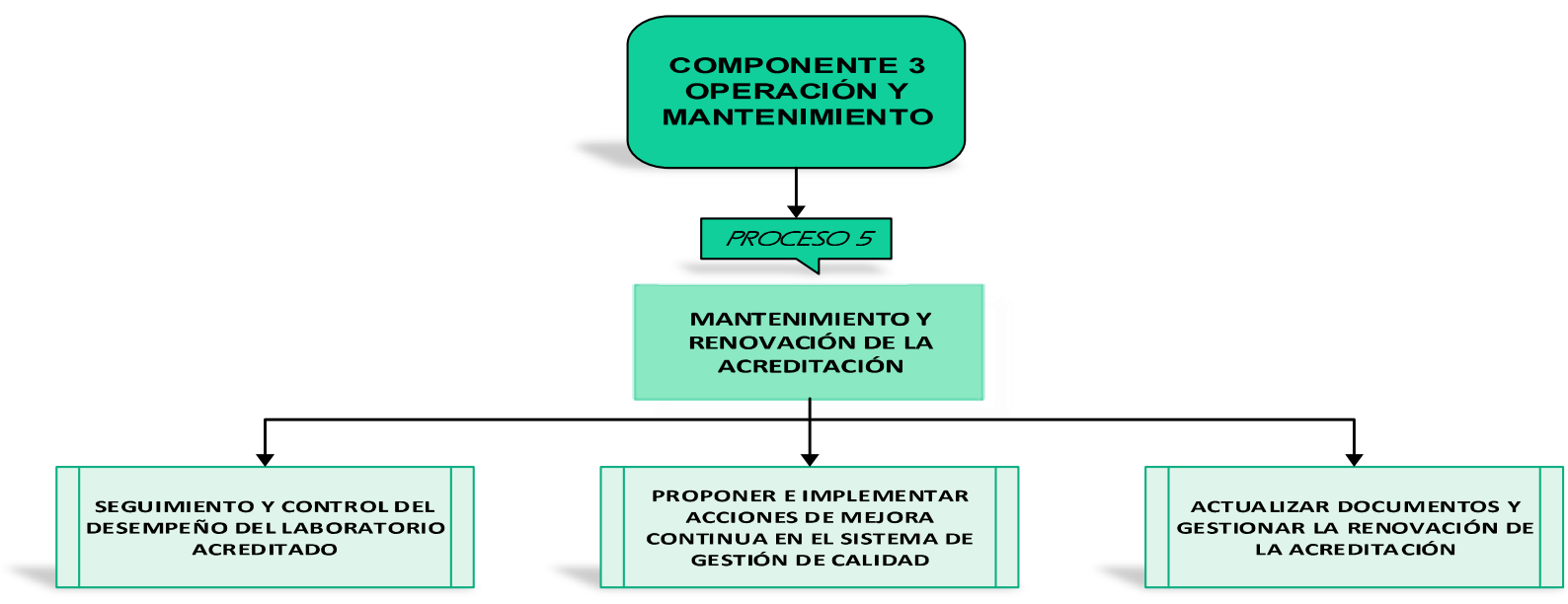

Figura 4. Componente 3: Operación y mantenimiento

Todos los procesos están contemplados en documentos anexos para su verificación y más amplio análisis.

Para la validación de la propuesta se realizó una segunda encuesta, esta vez sólo al grupo de expertos con una escala de valoración de 3 si es alto, 2 si el grado de validez se considera medio y uno si se considera un grado de validez bajo y se presentan en la tabla 1.

Tabla 1

Resultados de la aplicación de los procesos de la Metodología de Gestión Operativa propuesta.

Dimensión metodológica

Proceso 0: Diagnóstico Situacional

Proceso 1: Procesos de inicio

Proceso 2: Procesos de planificación

Proceso 3: Proceso de ejecución monitoreo, control.

Proceso 4: Proceso de cierre

Proceso 5: Proceso de seguimiento y control
Grado de validez

Alto

Alto

Alto

Alto

Medio

Alto

El nivel de validez de la metodología de implementación de la Norma NTP ISO/IEC 17025:2017 propuesta, es alta; por lo tanto, constituye una alternativa viable para la solución del problema de investigación, según los expertos. Considerando los resultados de los análisis de la opinión de expertos, se determina que la metodología de implementación propuesta, constituye una alternativa viable para la acreditación de los laboratorios de ensayo de suelos, concretos y pavimentos en Universidades Privadas del Perú. 


\section{DISCUSIÓN}

Se desarrolló una metodología de implementación de la Norma NTP-ISO/IEC 17025:2017 para la acreditación de laboratorios de ensayo de suelos, concretos y pavimentos en Universidades Privadas del Perú. Se determinó que, en los laboratorios de suelos, concretos y pavimentos de las universidades privadas del Perú no está implementada la Norma NTP-ISO/IEC 17025:2017, actualmente sólo un laboratorio de una Universidad Privada se encuentra acreditada para ejecutar un ensayo vinculado al rubro de la construcción (concreto). Es muy importante la acreditación de los laboratorios de ensayo vinculados al control de calidad de obras, con la finalidad de reducir la informalidad y mejorar el posicionamiento de nuestro país en cuanto a calidad de la infraestructura. Para el desarrollo se utilizó la Metodología del PMBOK (Project Management Body of Knowlegde), estándar de gestión de proyectos gestionado por el Project Management Institute (PMI). Esta metodología es reconocida internacionalmente, pues brinda una guía con una serie de prácticas que se pueden adaptar a las diversas necesidades de un proyecto, la sexta edición de la Guía del PMBOK está vigente desde el año 2017, y está estructurada en cinco grupos básicos de procesos, diez áreas de conocimiento y cuarenta y nueve procesos.

Se propone los procesos y procedimientos de los componentes de la metodología de implementación para la acreditación de laboratorios de ensayo conforme a los requisitos que exige la Norma NTPISO/IEC 17025:2017. Se validó con expertos que la metodología propuesta de implementación de la Norma NTP ISO/IEC 17025:2017 es una alternativa viable para la acreditación de laboratorios de mecánica de suelos, concretos y pavimentos en Universidades privadas del Perú.

Los resultados se encuentran en concordancia con lo propuesto por Uribe, M. (2011 que presenta una reseña de 32 textos relacionados con el tema, que es una herramienta muy útil para el caso de investigación. Sagrado, S., Bonet, E. \& Medina, M. (2017), que desarrolla el Manual práctico de calidad en los laboratorios: enfoque ISO 17025. Contreras, H., \& Galleguillos, L. 2009, presenta pautas importantes para normalizar un laboratorio de ensayos en maderas utilizando la Norma ISO 17025. Quintela, M. (2015) que encuentra herramientas útiles para aumentar la eficacia de un laboratorio, garantizar la fiabilidad de los resultados y reducción de costos. Ramírez, M. \& Flores, A. (2010), presenta un amplio enfoque referente a la normalización, las normas son el fundamento de la calidad. Escamilla, A. (2014) que resume los principios de operación, calibración, verificación de diversos instrumentos de medición.

Se recomienda que las universidades privadas del Perú tomen en cuenta la presente metodología validada por expertos como una alternativa para acreditar bajo los estándares de la Norma NTP/ISO IEC 17025:2017. Las universidades nacionales interesadas en acreditar también podrían considerar la metodología propuesta según los requisitos de la Norma NTP ISO/IEC 17025:2017 para la acreditación de sus laboratorios. La presente metodología también es aplicable a laboratorios de otras especialidades, por ello se recomienda a las instituciones universitarias trabajar con la presente metodología propuesta para efectos de comprobar la validez de sus procesos según los requisitos de la Norma NTP ISO/IEC 17025:2017.

\section{REFERENCIAS BIBLIOGRÁFICAS}

Ackerman, S., \& Com, S. (2013), Metodología de la investigación.

Brache, A., \& Bodley, Sam. (2006), Implementación: cómo transformar las iniciativas estratégicas en resultados.

Contreras, H., \& Galleguillos, L. (2009), Pautas para la normalización de las actividades del Laboratorio de ciencias y evaluación de tratamientos del departamento de ingeniería de maderas.

Córdova, M.(2012), Plan para la adecuación del Laboratorio de ensayo de materiales (LEM-FIC-UNI) a la Norma Internacional ISO/IEC 17025.

Díaz, M., \& Araya, M. (2007), Propuesta metodológica para obtener la acreditación del área de operaciones de un laboratorio de análisis de aguas según NCH ISO 17025. 
Diccionario ilustrado de la Lengua Española.

Escamilla, A. (2014), Metrología y sus aplicaciones.

López, P. (2015), Cómo documentar un sistema de gestión de calidad según ISO 9001:2015.

Marcelino, M., \& Ramírez D. (2014), Administración de la calidad: nuevas perspectivas.

Norma NTP-ISO/IEC 17025:2017, Requisitos generales para la competencia de los laboratorios de ensayo y calibración.

Norma NTP-ISO/IEC 17025:2006, Requisitos generales para la competencia de los laboratorios de ensayo y calibración.

Núñez, E. (2007), Archivos y Normas ISO.

Quintela, M. (2015), Diseño de test de cualificación OQ y PQ en HPLC y desarrollo de modelos de cálculo de la incertidumbre a partir de la validación de procedimientos de análisis.

Ramírez, M. \& Flores, A. (2010), Metrología y Normalización.

Sagrado, S., Bonet, E. \& Medina, M. (2017), Manual práctico de calidad en los laboratorios: enfoque ISO 17025.

Tejerina, M. (2010), Propuesta de implementación de la ISO/IEC 17025 para mejorar los servicios del Laboratorio CITELAB Tacna-2010.

Uribe, M. (2011), Los sistemas de gestión de la calidad: el enfoque teórico y la aplicación empresarial.

World Economic Forum (2017) Informe Global de Competitividad (ICG) 2017-2018. 